\title{
Entre antropología y literatura: Recepción de El umbral roto. Escritos en antropología poética*
}

\author{
Between anthropology and literature: Reception of \\ El umbral roto. Escritos en antropología poética
}

\section{Pilar Valenzuela Rettig **}

Universidad Austral de Chile. Valdivia, Chile

valenzuela.pilar@gmail.com

\section{RESUMEN}

En este trabajo se presenta y analiza la recepción del texto El umbral roto. Escritos en antropología poética del autor Juan Carlos Olivares. Se postula que las recepciones de la obra y de la antropología poética, textualidad instaurada por la obra de Olivares, van desde el campo antropológico al interdisciplinario. Para ello se analizan las recepciones realizadas a nivel paratextual, la recepción como antropología poética, mutación disciplinaria y literatura antropológica.

Palabras clave: Antropología poética, mutación disciplinaria, literatura antropológica en Chile, Juan Carlos Olivares, recepción.

\section{ABSTRACT}

This paper presents and analyzes the text reception El umbral roto. Escritos en antropología poética of Juan Carlos Olivares. It is postulated that the reception of the

* Este artículo forma parte del Proyecto Fondecyt Regular 2010 No1100344 "Literatura antropológica en Chile”, investigador responsable Dr. Iván Carrasco. Se realizó a partir de la ponencia del mismo nombre presentada en el XVII Congreso Internacional de Estudios Literarios, UPLA, Valparaíso.

"Tesista del Programa de Doctorado en Ciencias Humanas, Mención Discurso y Cultura, de la Universidad Austral de Chile. Becaria CONICYT. 
work and anthropology poetic textuality established by the work of Olivares, ranging from anthropology to interdisciplinary field. To do this, we analyze the paratextual reception held at the reception as poetic anthropology, disciplinary mutation and anthropological literature.

Keywords: Anthropology poetic, disciplinary mutation, anthropological literature in Chile, Juan Carlos Olivares, reception.

Recibido: 02.08.2012. Aceptado: 03.10.2012.

\section{INTRODUCCIÓN}

Fl umbral roto. Escritos en antropología poética es un texto del antropó$\angle$ logo Juan Carlos Olivares, publicado en 1995 por el Fondo Matta y el Museo Chileno de Arte Precolombino. A través de este texto, Olivares presenta el concepto de antropología poética para referirse a su quehacer escritural, caracterizada como una forma de escribir un trabajo antropológico, una etnografía, con un lenguaje cuidado, poético, que se escapa de los cánones del trabajo científico.

A primera lectura, El umbral roto... es un texto híbrido, al presentar características literarias y antropológicas. La escritura de Olivares se debate entre lo poético y lo antropológico a través de la narración, la prosa poética, las citas textuales, las referencias a antropólogos, filósofos y escritores literarios, entre otros recursos de ambas disciplinas. Por lo tanto, podemos considerar que presenta elementos de ambos campos disciplinarios, entendiendo este concepto como un campo de fuerzas que actúan sobre todos aquellos que entran en él, y de manera diferente, dependiendo de la posición que los sujetos ocupan en el campo; un campo sustentado en competencias y tomas de posiciones que se generan en un conflicto permanente (Bourdieu, 1993: 323).

El umbral roto... puede considerarse una obra clave de la textualidad denominada antropología poética. Esta se caracteriza por ser un conjunto de textos creados por antropólogos, difundidos en congresos y editoriales alternativas, no en un marco de publicaciones científicas o de antropología clásica, racionalista. La antropología poética es una textualidad poco estudiada, que se ha ido instituyendo en un campo disciplinario y un régimen discursivo ambivalente; es decir, la antropología poética posee una localización paradójica debido a la escasa legitimación y regulación de la producción y consumo de las obras. Como bien lo expresa su nombre, es una 
textualidad que se alimenta de la antropología y la literatura. ¿Es un tipo de antropología, de literatura? ¿Es propia de ambas disciplinas o de ninguna? El presente artículo busca constituir un aporte al problema que generan estas interrogantes, a través del análisis de la institucionalidad de ésta, a partir del estudio de la recepción del texto que instaura este concepto.

El objetivo del artículo es analizar la recepción de la obra, sobre todo a partir de los paratextos que la acompañan en el libro (1995), la tesis doctoral de Miguel Alvarado (2001) y la valoración que realiza Iván Carrasco bajo las concepciones de mutación disciplinaria (2003) y con Alvarado de literatura antropológica (2010).

La hipótesis que guía el presente estudio es que la recepción de la obra ha sido realizada desde el campo antropológico al interdisciplinario; primero, bajo el concepto de antropología poética o mutación disciplinaria, para llegar a la recepción como una forma de literatura antropológica.

\section{LOS PARATEXTOS. PRIMERA RECEPCIÓN DE LA OBRA}

La primera recepción que vamos a estudiar es la realizada en torno a la presentación de la obra en el libro, sobre la base de los paratextos que indican el modo de concebirlos por parte de quienes opinan sobre él. Estos paratextos -entendidos como conjuntos de signos mediante los cuales un texto se hace libro y se propone como tal a sus lectores; signos que bordean o prolongan al texto para presentarlo (Genette, 1992: 7-8)- determinan la lectura del texto y corresponden a: dos solapas, una bajo la autoría de Francisco Gallardo Ibáñez, del Museo Chileno de Arte Precolombino, otra anónima y tres textos agrupados bajo el título de "sumario": "Presentación" de Carlos Aldunate del Solar, Director del Museo Chileno de Arte Precolombino, "Atacama off line...” de Francisco Gallardo, adscrito al museo, y "El oficio del etnógrafo y la etnografía como artificio: reflexiones y presunciones" de Daniel Quiroz.

La primera solapa corresponde a un escrito en primera persona de Francisco Gallardo. A través de este paratexto conocemos la opinión de un antropólogo que se adscribe a una de las instituciones que hace posible la publicación del texto de Olivares: el Museo Chileno de Arte Precolombino. Se expresa el conocimiento de los textos antes de su publicación y se enfatiza la capacidad de involucrar al lector en los ambientes descritos; luego, su pensamiento con respecto a que en la actualidad estos textos son la mejor invitación para "caer en el vacío y la oscuridad donde podría revelarse el secreto de lo oculto". Cabe destacar la intención poética de estas citas: se 
comenta el texto de Olivares con un lenguaje que se acerca más a lo poético que a lo científico, un lenguaje propio de una crítica literaria, no de una reseña de un texto antropológico.

En la solapa de contraportada se presenta un paratexto sin autoría que se refiere al autor del libro. Primero se hace referencia a "Juan Carlos Olivares Toledo, visto por él mismo" en cuanto a poesía, estudios, antropología y la relación que establece con Daniel Quiroz, basada en la amistad y el aprendizaje (se refiere a Quiroz como su maestro), y el amparo de "su antropología" en los espacios de la Dirección de Bibliotecas, Archivos y Museos, para terminar la presentación del autor como el que dirige el "museoazul de las islas de Chiloé” (el Museo Regional de Ancud). Este paratexto dirige la recepción del texto hacia el autor antropólogo.

La primera sección del conjunto titulado "sumario" se concibe bajo la autoría del director del Museo Chileno de Arte Precolombino y corresponde, como lo expresa su título, a la presentación formal por parte del museo. En ella se expresa que el museo "ha encontrado un lugar en el acogedor seno de las ciencias antropológicas y por ello no deja de estar atento ante sus múltiples desarrollos" (Olivares, 1995: 7), dejando entrever que el texto corresponde a una forma más de desarrollo antropológico. En el siguiente párrafo expone que la antropología ha presentado un "giro poético" (el concepto se presenta entre comillas), queriendo enfrentar la "objetividad" (nuevamente el concepto entre comillas) del antropólogo con sus vivencias etnográficas personales, con un tipo de expresión "que nos introduce de lleno en el campo de la metáfora y la poética, y que se inscribe en un mundo literario anunciado por Lévi-Strauss en su obra Tristes Trópicos" (Olivares, 1995: 7). A través de la relación que se establece entre el texto y la obra literaria-poética de Lévi-Strauss, se inscribe a El umbral roto... en este linaje en desarrollo que "anuncia" el célebre antropólogo. El párrafo que continúa, el último, expresa que la antropología poética crece en el amparo del museo gracias a la colaboración del artista Roberto Matta y que esperan contribuir a un acercamiento de la ciencia hacia el arte.

En esta "presentación”, a través de un discurso formal, se contextualiza la producción de la obra y del "giro poético" de la antropología. Se expresa una recepción del texto bajo el concepto que él mismo propone: Antropología Poética y se presenta como una línea de desarrollo más de la antropología, como una forma de etnografía que se "inscribe en el mundo literario" (1995: 7), como una "experiencia" que pretende unir ciencia y arte. Así se inserta el texto en el campo antropológico y su "expresión" en el literario.

El siguiente paratexto, Atacama off line... debería corresponder a una 
recepción, sin embargo, da la impresión de que el texto de Francisco Gallardo pretende ser una obra de antropología poética, puesto que presenta una temática antropológica, un discurso con características etnográficas: la experiencia personal de viaje a Atacama y sensaciones en relación a esta experiencia. Introduce dos párrafos entre paréntesis: uno que corresponde a una anotación de un cuaderno de campo, y otro que corresponde a una cita de un diccionario. Sólo en el último párrafo hace referencia expresa al texto de Olivares. El párrafo dice: "El sueño de esta antropología/poética que nace no es grave, es por humor. No quiere olvidar el juego o creer que todo está perdido" (9; el subrayado es nuestro). Interpretamos que Gallardo recepciona la obra como antropología poética, entendiendo a ésta como una forma de hacer etnografía de manera lúdica, así como él presenta lúdicamente el texto.

El último paratexto de "sumario" corresponde al antropólogo Daniel Quiroz, quien es presentado en la solapa de la contraportada como amigo y maestro de Olivares. A partir de esta autoría podemos establecer una recepción que va más allá de la lectura, puesto que es evidente una relación con el autor que establece un conocimiento profundo del proyecto escritural y los pormenores presentes en el proceso de construcción textual. Esta interpretación se refuerza con el inicio del paratexto "El oficio del antropólogo y la etnografía como artificio: reflexiones y presunciones", al expresar que "escribir estas líneas debe se una de las cosas más queridas y por eso ha sido de las más difíciles. Desde hace más de diez años hemos construido con J.C. Olivares relatos y hemos aprendido uno del otro a crear nuestras propias formas de antropología” (11). Esta recepción se basa en la amistad y en el compañerismo en el definir un "nosotros antropólogos"; es decir, corresponde a una recepción desde el campo de la antropología y, en específico, un antropólogo que comparte con el autor sus "propias formas de antropología”.

Quiroz basa su presentación en el tema de la etnografía. Considera que la obra de Olivares es "un punto sobre el que apoyarnos para reflexionar sobre el significado del hacer etnografía (encontrarse) y del escribir etnografía (alejarse)" (11). Desde esta afirmación parte la reflexión en torno al ejercicio de la etnografía, el encuentro etnográfico, el viaje, el trabajo de campo, la situación etnográfica, concluyendo: "El oficio del etnógrafo se aprende en la práctica del trabajo de campo, nunca en la sala de clases" (15); "La etnografía es un bien escaso, todavía muy escaso" (15); y "la etnografía no sólo es experiencia sino también escritura. La construcción del relato etnográfico es tal vez la tarea más descuidada de la antropología" (15), considerando que Olivares demuestra un estilo "inusual" entre los escritores de antropología, 
ante lo que afirma más adelante: "Olivares ha señalado que siempre ha querido escribir poéticamente la etnografía” (16). Finaliza su reflexión con una definición de etnografía poética:

Una etnografía poética no es sino crear la descripción del encuentro con la imaginación y rigurosidad que nos exige la poesía. La poesía nos permite transmitir las emociones y sensaciones que nos provoca el encuentro etnográfico. Muchos antropólogos han escrito sobre las relaciones entre poesía y etnografía [...] No quiero hacerlo pues me parece que no es posible, aunque a veces debemos intentar la imposible (16).

De la cita se extrae un conjunto de opuestos que adscriben al texto de Olivares al campo literario y antropológico: imaginación/rigurosidad, emociones-sensaciones/encuentro etnográfico. Estos opuestos son sintetizados mediante la fórmula "escribir poéticamente la etnografía", presentada en la cita anterior.

Quiroz establece un acercamiento al concepto de antropología poética previo a la lectura del texto, basándose en que ésta es una forma de escritura poética del encuentro etnográfico; es decir, la sitúa en el campo antropológico, pero con una forma poética de expresión.

Luego de esta "conceptualización” de antropología poética, Quiroz se refiere a la recepción de los textos antropológicos: "Nuestros textos tienen un destino pues están escritos para ser leídos. Escribimos para nuestros lectores" (16), ubicando así su recepción como la de un antropólogo que comparte el oficio de la escritura con el autor del texto. Y continúa: "Tengo la sospecha que, de una cierta manera, Olivares escribe para sus amigos, con los que comparte algunos secretos, algunas claves. Somos una parte de su auditórium y este libro representa un esfuerzo por ampliarlo a un grupo más grande de personas" (16).

Finaliza su texto preguntándose si "basta el oficio de escribir para ser un buen etnógrafo" (17) y afirmando que Olivares es un maestro en este oficio. Llama a la lectura en busca de la "historia perdida", invitando a leer estos "textos escritos con dolor, rocío y ternura, palabras que son etnografía pura" (17): nuevamente destaca un lenguaje poético que transmite la empatía con la carga emotiva que transmite el texto de Olivares.

En resumen, Quiroz, en El oficio y la etnografía como artificio: reflexiones $y$ presunciones, presenta opinión desde el campo de la antropología, determinada por una relación de amistad y empatía con el autor del texto. Determina el texto como una forma de etnografía llamada antropología poética, 
y que la poesía corresponde a una forma de escritura que permite transmitir emociones y sensaciones provocadas por el encuentro etnográfico.

\section{RECEPCIÓN DE LA OBRA COMO ANTROPOLOGÍA POÉTICA CHILENA}

Miguel Alvarado, en su tesis doctoral La antropología poética chilena como textualidad híbrida (2001), patrocinada por el profesor Iván Carrasco, comenta el texto El umbral roto... como uno de lo precursores de la antropología poética chilena.

Alvarado afirma que "el conjunto de obras compuesto por la 'Antropología Poética' consiste en un tipo de producción textual iniciadora de un nuevo género discursivo, en tanto no responde pragmáticamente ni al canon científico ni al literario con exclusividad" (319). Afirma que estas obras generan sus propios circuitos de circulación, recepción y lectura. Son los espacios institucionales y culturales, como el Museo Chileno de Arte Precolombino, el Fondo Matta, el Departamento de Antropología de la Universidad de Chile, el Museo Regional de Ancud y diversos proyectos FONDART y CONICYT, los que constituyen espacios de producción de estos textos, aunque marginalmente, debido a que estas obras se consideran experimentos de nuevas formas de discurso (319).

En cuanto al carácter literario de la "Antropología Poética", Alvarado sostiene que la literatura se encuentra excluida de la metalengua explícita e implícita de los textos, en tanto "no hay intención de moverse pragmáticamente en el ámbito literario de parte de sus autores y menos aún se intenta que los textos sean recepcionados como literarios" (319); no obstante, sostiene que este nuevo género "interconecta el uso de elementos macroestructurales, superestructurales y de tipos de discurso, formas intertextuales (desde la literatura hacia el texto antropológico literario) y textos metalingüísticos, propios de los géneros literario y científico" (320), configurando una identidad genérica que supera el plano de lo pragmáticamente funcional. Concluye que este conjunto textual, llamado por él "Antropología Poética Chilena", es, básicamente, "un nuevo subgénero antropológico, su relación con la literatura tiene un carácter instrumental' (324, el subrayado es nuestro). Afirma que "la literatura confiere una base expresiva a esta antropología poética en lo que respecta al nivel transtextual, a la generación de superestructuras textuales y tipos de discurso, y a la metalengua presente en la corriente" (320). Sin embargo, Alvarado concluye que sus macroestructuras responden 
al modo antropológico de producir un texto, centrándose en la temática de la diversidad cultural; no obstante, en las mismas conclusiones afirma que estas macroestructuras textuales, que corresponden a problemáticas de carácter antropológico, problemas científicos, "ya han sido tratados desde una óptica literaria y con ello se suscita un proceso fundamental de recurrir a la literatura como fuente expresiva" (321).

El autor concluye que la base antropológica de este nuevo género textual, correspondiente a la macroestructura de carácter antropológico, "se ve reafirmada en el modo que desde la pragmática se ha definido en cada uno de los textos, los que circulan eminentemente en los circuitos antropológicos, ninguna de estas obras son dirigidas a un público masivo" (321).

A partir del análisis de los textos, Alvarado (2001: 324-326) concluye en su tesis que éstos presentan como punto de encuentro características que le confieren identidad a la corriente antropológico-poética: el tema de la diversidad cultural como macroestructura textual; el autor modelo de los textos presenta como afirmación básica la crítica de los límites del discurso antropológico tradicional y la necesidad de innovar la textualidad para dar cuenta de las distintas expresiones de diversidad sociocultural, tanto en el ámbito teórico como etnográfico; el lector modelo presenta una clara postura de abertura a la innovación textual, capaz de aceptar y legitimizar esta postura, ubicándola en el contexto intelectual chileno; la metalengua implícita y/o explícita responde a la lógica según la cual el punto de partida de la creación es la constatación de los límites del discurso antropológico en cuanto a capacidad de expresión de la diversidad y las emociones que dicha diversidad provoca en el autor; una constante transtextualidad que recurre a la literatura para complementar las formas de expresión y los conceptos propios del discurso antropológico.

En particular, Juan Carlos Olivares es considerado por Alvarado (2001: 189-295) como uno de los precursores de la antropología poética. Considera que con el texto El umbral roto... Olivares presenta dos hitos fundamentales para la conformación de la corriente: la expresión metalingüística de la hibridez estructural, asumida como una propuesta productiva y la propuesta del concepto de "Antropología Poética" (189). Reconoce los siguientes aspectos en su análisis del texto: la situación que enmarca la creación del texto, el nihilismo y la opción ética que presenta, la tendencia hermenéutica, el concepto de poesía presente, el uso de la metáfora e ironía, caracterización del lector modelo, la transtextualidad y la autorreferencia como tema.

En cuanto al concepto de poesía presente, Alvarado lo ubica en un par binario y tensionado entre poesía (arte)/antropología (ciencia) que confi- 
guran la hibridez de esta modalidad discursiva (193). La poesía opera, en un primer nivel, como plano desde donde se cuestiona el canon antropológico y, en segundo nivel, determinado por el uso de figuras retóricas, determina lo novedoso del género textual (193). Sostiene que existe un intento de ser coherente con la antropología y una situación marginal respecto de los círculos literarios. Sin embargo, no afirma que el lector modelo corresponda a un antropólogo, sí que éste, como estrategia textual, no tiene antecedentes. Se piensa en un lector crítico en extremo, ante todo, con la antropología, a lo menos con la antropología de la década de los 80 en Chile y anterior de Europa (194).

La literatura es considerada como "un tipo de personaje más en la polifonía de Olivares" (198). La transtextualidad la determina por la presencia de la literatura, que "irrumpe como un soporte" (198), y la antropología que "como fuente es un invitado transtextual tímido, un tanto mutilado. Se nos presenta como un discurso que se pregunta por sí mismo, cada vez más consciente de sus limitaciones" (198).

\section{RECEPCIÓN COMO MUTACIÓN DISCIPLINARIA}

Iván Carrasco (2003) publica el artículo "La antropología poética como mutación disciplinaria". El autor presenta algunas observaciones críticas, desde una perspectiva semiótica, de la antropología poética, bajo la hipótesis de que ésta "constituye una textualidad multifacética que desarrolla un tipo de discurso contemporáneo caracterizado por la "mutación disciplinaria" (7). En dicho artículo se presenta un análisis del texto El umbral roto... a manera de ejemplificación de sus postulados.

Carrasco recepciona el texto como antropología poética, aceptando la postura del autor, al igual que las recepciones anteriores. Con respecto a este conjunto textual expresa:

La antropología poética chilena consiste básicamente en enfatizar una dimensión del trabajo etnográfico, la escritura, es decir, la construcción de relatos distintos a los de la tradición etnográfica inmediata, europea y norteamericana, representando una reacción sui generis ante la crisis del conocimiento científico racionalista derivado de la fuerte proyección de las ciencias de la naturaleza en otros campos. La preocupación por el texto y su metalengua acerca a los antropólogos chilenos jóvenes a los poetas y novelistas, quienes pasan a constituir modelos de estilo y, en cierta medida, 
también formas de vida, es decir, presentándose como héroes intelectuales (15).

Manifiesta coincidir con la postura de Alvarado en la opinión de que la antropología poética no es específicamente una nueva forma de literatura, puesto que la metalengua de los textos tiende a apartarse del canon literario y no existe una intención de recepción literaria (8). Considera que esta metalengua tampoco coincide del todo con el canon antropológico científico positivista, al resentir el concepto de ciencia y la posibilidad de acumular conocimiento.

Carrasco explica esta manifestación textual desde la mirada de "mutación disciplinaria", entendida como: "la modificación de reglas, materias y modalidades de conformación de textos de una disciplina artística, científica o filosófica, provocada por el traslado desde una u otra disciplina de la misma o distinta condición" (8). Bajo esta postura se recepciona el texto y la antropología poética no como antropológico o literario sino como un tipo textual híbrido fundado en el artículo científico y en el texto literario. Es decir, Carrasco considera que El umbral roto... es una textualidad que proviene de la antropología, pero que adquiere hibridez debido a la modificación de reglas, materias y modalidades, propias del campo antropológico por reglas, materias y modalidades literarias.

Los autores, considera Carrasco, son antropólogos que pretenden e intentan escribir como poetas; "intuyeron o aprendieron que la literatura también promueve una intensa necesidad de discurso de lo real, de apertura al mundo, el conocimiento y los lenguajes, junto a sus tradicionales recursos retóricos y estilísticos que le otorgan su elevada capacidad de convicción" (9). Sin embargo, la condición multifacética de su discurso se encuentra limitada por dos discursos conductores, el antropológico y el literario, inclinándose hacia el antropológico, a pesar de intentar mantener el equilibrio (10). Más adelante expresa que los autores "tratan de aprehender las cualidades propias de la poesía (predominio de la enunciación o del sujeto, libertad de elección de referencias empíricas, imaginarias, emotivas, verbales, etc.), pero sin dejar de ser antropología" (12).

Carrasco ejemplifica estos y otros postulados, con la obra en estudio. Expresa que El umbral roto... es el texto fundador de la tendencia (13). Analiza semióticamente el título, manifestando que éste es de carácter mixto: la primera sección en lenguaje metafórico, de uso preferentemente poético, y la segunda corresponde a una frase o fase? denotativa propia del lenguaje científico, interpreta que "la oscilación entre estos dos polos representa la 
ambigüedad disciplinaria del trabajo propuesto por Olivares, antropología (pero no científica sino poética), oposición o paradoja, según desde donde se mire" (13.)

Considera que el discurso es eminentemente subjetivo, aunque también habla de personajes empíricos, de paisajes, objetos, ideas, teoría. Es heterogéneo, ya que está formado por una variedad de citas (etnográficas, literarias, filosóficas, etc.), recuerdos, proposiciones teóricas, etc. Considera que su propósito es "buscar una manera diferente de escribir el relato etnográfico, preocupación de escritor en vez de teórico o investigador, por lo cual se acerca mucho más al relato testimonial y al poema en prosa, géneros característicos de la literatura" (13). Por lo tanto, expresa Carrasco, "se presenta equívocamente como reflexión antropológica, se convierte parcialmente en literario, distorsionando los tipos de discurso, mezclando los estilos, transformando el objeto de estudio y el modo de vincularse con la realidad observada mediante la intuición poética” (13).

Sintetizando, Carrasco recepciona la obra como el texto fundador del tipo textual llamado antropología poética, tal como lo propone el autor. Coincide con Alvarado en determinar que no corresponde a una modalidad textual literaria, pero no en que sea un tipo de antropología, sino un tipo de mutación disciplinaria. De esta manera, ubica el texto en un espacio ambiguo, que podría llamarse "campo interdisciplinario", donde confluyen elementos antropológicos y literarios. A pesar de esta ambigüedad, considera que el texto, la antropología poética, tiende más hacia la antropología.

\section{RECEPCIÓN COMO LITERATURA ANTROPOLÓGICA EN CHILE}

Carrasco en el 2010 presenta el artículo "Literatura antropológica chilena: fundamentos" en Estudios Filológicos. En este texto se presenta la base teórica de esta nueva literatura chilena y los aspectos generales del proyecto. Considera que El umbral roto... conforma parte del corpus de esta textualidad (22).

Según el autor, la literatura antropológica chilena es:

${ }^{1}$ Este artículo forma parte del Proyecto Fondecyt Regular "Literatura antropológica en Chile". Consideramos el artículo como autoría de Carrasco por ser él quien instaura el concepto de esta literatura, a pesar de que la publicación la realiza con Alvarado, el que se especializa en "antropología literaria”. 
(...) un tipo de género discursivo, y quizás también una tendencia literaria, que aún no ha sido conocido, valorado, leído ni interpretado como tal, es decir, no ha sido canonizado por la institución literaria porque se ha desarrollado básicamente en publicaciones, congresos y diálogos propios de los espacios intelectuales de un sector determinado de las ciencias sociales contemporáneas (9-10).

\section{Y más adelante:}

Se trata de un conjunto de textos literarios de carácter principalmente etnográfico, escritos por antropólogos y arqueólogos contemporáneos, textos mediacionales en busca de nuevas experiencias y modos de conocimiento, como la intuición creadora del poeta o novelista. Esta literatura ha surgido en medio de la escritura antropológica y puede considerarse un sector de la literatura chilena por su parcial analogía temática y estilística con la textualidad literaria moderna y posmoderna, pero al mismo tiempo no ha perdido sus cualidades etnográficas y etnológicas (14).

Es así como se refirma que esta textualidad es una modalidad de mutación disciplinaria, donde se remite principalmente a las órdenes del pensamiento literario y antropológico (10 y 14). Con respecto a la literatura, y al inicio de ésta en particular, recuerda que esta ciencia es una de las actividades humanas que se transforma con mayor rapidez en cuanto actividad creativa y en cuanto a sus productos debido a la búsqueda permanente de originalidad, y considera que tal vez por esta razón ha pasado desapercibida esta tendencia literaria cuyo origen fija en 1995, año de la publicación del texto en estudio (12).

Determina como antecedentes principales de esta literatura a la antropología poética y literaria (14), considerando como parte de la presentación teórica de estas antropologías, los estudios de Alvarado y el propio (2003). Considera que Alvarado y otros, como Cárcamo (2007) y González (2003), han ubicado a esta/s textualidad/es en el campo de la antropología; considerándose como subáreas de la historia del pensamiento antropológico, por tanto, forman parte de los intentos de renovación de la antropología y no pueden considerarse "literarias" en sentido estricto, sino aproximaciones interdisciplinarias a la literatura (15). Frente a éstas, considera que la literatura antropológica posee un estilo propio, un conjunto de temas condicionados por formación profesional de los autores, una composición que se acerca al ensayo literario, adoptando principalmente la forma de collage o texto 
fragmentario. Expresa que "lo literario" se ha leído como tal mediante tipos de textos ya consagrados como literarios, permitiendo que personas de otra formación, no literaria, como algunos antropólogos hayan transformado sus textos en busca de producción de textos literarios que ofrecen una experiencia distinta a la científica, quizás, según el autor, mayor intensidad, libertad y acceso a la trascendencia (15-16). Con respecto a la calidad literaria de estos textos, reconoce que hay textos de mejor o peor calidad literaria que otros y que en ocasiones es difícil reconocerla, pero que esta situación también se da en la escritura literaria habitual (17).

Si bien en este artículo se presenta la base teórica de la literatura antropológica en Chile, no se presenta un análisis del texto de Olivares. La recepción se considera en el hecho de presentar el texto en estudio como parte del corpus de la literatura antropológica en Chile, como antes lo ha realizado Alvarado en su tesis doctoral y el mismo Carrasco en su artículo sobre antropología poética como mutación disciplinaria.

\section{CONCLUSIONES}

La obra de Juan Carlos Olivares, El umbral roto. Escritos en antropología poética, fue publicada en 1995 por el Fondo Matta y el Museo de Arte Precolombino; siendo, de esta manera, instaurada en un campo antropológico, determinando una recepción textual antropológica que se sostiene, además de su medio de publicación, por los paratextos. Estos corresponden a recepciones realizadas por miembros del campo antropológico cercanos al autor y a la edición del libro: miembros del Museo y el antropólogo Daniel Quiroz, amigo y compañero de oficio, más aún, compañero de la "mirada antropológica alternativa” que presenta Olivares. A través de estos paratextos se legitima el concepto que Olivares propone para denominar su quehacer antropológico: antropología poética. La recepción paratextual sostiene el concepto mediante un marco conceptual amplio, como es el giro poético y al sostener que la antropología poética que propone Olivares corresponde a uno de los múltiples desarrollos de la antropología.

En el año 2001 concluye la tesis doctoral de Miguel Alvarado, ofreciendo el estudio más profundo sobre la antropología poética. Alvarado sostiene que la obra de Olivares instaura el concepto y presenta por primera vez una metalengua en relación a la antropología poética. A partir de esta obra surgen una serie de textos creados por antropólogos chilenos que se inscriben en esta textualidad. 
Alvarado recepciona la obra bajo el concepto de antropología poética chilena, como texto precursor de un nuevo género discursivo, que no corresponde al canon antropológico ni al literario. Sin embargo, es concluyente al calificarlo como un "nuevo subgénero antropológico", determinando que las características literarias quedan sujetas al orden instrumental y expresivo.

Consideramos que la recepción de Alvarado también se inscribe dentro del campo antropológico. Hay que considerar que Alvarado es antropólogo de profesión y, a pesar de presentar una investigación de carácter interdisciplinaria, se basa en un marco teórico débil en aspectos de teoría literaria.

Posterior a la tesis de Alvarado, su profesor guía, Iván Carrasco, publica el artículo La antropología poética como mutación disciplinaria (2003). Carrasco va más allá de la recepción "antropológica" realizada previamente, ubicando a la antropología poética y la obra en estudio como exponente de una manifestación interdisciplinaria denominada "mutación interdisciplinaria”.

Carrasco realiza una serie de observaciones semióticas ejemplificando con el texto de Olivares. Concuerda con Alvarado en el hecho de sostener que esta textualidad no es un nuevo tipo de literatura debido a la metalengua presente en el texto y en el resto del corpus de la antropología poética. Se expresa que en la metalengua de esta textualidad no existen elementos para considerarla literaria ni la intención de ser recepcionada o instaurada como literatura. Sin embargo, Carrasco no considera esta textualidad como antropológica, sino como un tipo textual híbrido fundado en el artículo científico y en el texto literario, con una inclinación hacia el antropológico.

En la actualidad se presenta la posibilidad de recepcionar la obra como literaria, bajo la postura teórica que presenta Carrasco al instaurar el concepto de "literatura antropológica en Chile" como una modalidad de mutación literaria y una nueva tendencia de literatura chilena. El autor considera el texto de Olivares como parte del corpus de esta literatura, a pesar de afirmar que la antropología poética y literaria son partes del desarrollo del pensamiento antropológico y diferente a la literatura antropológica. Pero esta situación de ambigüedad se supera al centrarse en la afirmación de que esta literatura es una modalidad de mutación disciplinaria y, por tanto, hay una situación de inter- $\mathrm{y}$ transdisciplinariedad.

Analizando la recepción de la obra y los fundamentos presentados por los estudios para "ubicar" la obra dentro de uno u otro espacio, antropología o literatura, concluimos que la hipótesis planteada se sostiene: la recepción va desde el campo antropológico al campo interdisciplinario. Las recepciones de los paratextos y de Alvarado se presentan desde el campo antropológico; 
la primera recepción de Carrasco se realiza desde el interdisciplinario bajo la postura de mutación disciplinaria, pero aún asimilando la obra como parte de la antropología poética; y, posteriormente, la recepciona como literaria, pero como parte del corpus de la literatura antropológica en Chile, una textualidad que se sostiene principalmente en la mutación interdisciplinaria.

\section{REFERENCIAS}

Alvarado, Miguel. (2001). La antropología poética chilena como textualidad híbrida. Tesis doctoral. Valdivia, Universidad Austral de Chile.

Bourdieu, Pierre. (1993). Les règles de l'art. Paris: Seuil.

Cárcamo, Solange. (2007). La antropología literaria: lenguaje intercultural de las ciencias humanas. Estudios Filológicos, 42, 7-23.

Carrasco, Iván. (2003). La antropología poética como mutación disciplinaria. Estudios Filológicos, 38, 7-17.

Carrasco, Iván y Alvarado, Miguel. (2010). Literatura antropológica chilena: fundamentos. Estudios Filológicos, 46, 9-23.

Genette, Gèrard. (1992). Seuils. Paris: Seuil.

González, Yanko. (2003). Nuevas prácticas etnográficas. El surgimiento de la antropología poética. En Nicolás Richard (comp.), Movimiento de campo, en torno a cuatro fronteras de la antropología en Chile (pp. 185202). Guatemala / Francia. Ed. ICAPI y Ecole des Hautes Etudes en Sciences Sociales.

Olivares, Juan Carlos (1995). El umbral roto. Escritos en antropología poética. Santiago: Fondo Matta y Museo Chileno de Arte Precolombino. 